\title{
RELATIVE TRACE FORMULA AND SIMPLE ALGEBRAS
}

\author{
YUVAL Z. FLICKER
}

\begin{abstract}
A Deligne-Kazhdan variant of the relative trace formula of Jacquet-Lai is given, thus generalizing the study of distinguished representations from the context of the quaternion algebra to that of any simple algebra.
\end{abstract}

1. Let $F$ be a global field, $E$ a separable quadratic extension, $\mathbf{A}=\mathbf{A}_{F}$ and $\mathbf{A}_{E}$ the associated rings of adeles, and $G$ a reductive $F$-group. Let $L(G)$ be the space of square-integrable functions $\varphi$ on $G(E) \backslash G\left(\mathbf{A}_{E}\right)$ such that for any proper parabolic subgroup $P$ of $G$ with unipotent radical $N$ we have $\int \varphi(n x) d n=0(n$ in $N(E) \backslash$ $\left.N\left(\mathbf{A}_{E}\right)\right)$ for any $x$ in $G\left(\mathbf{A}_{E}\right)$. An irreducible constituent of the representation $r$ of $G\left(\mathbf{A}_{E}\right)$ on $L(G)$ by right translations is called a cuspidal $G\left(\mathbf{A}_{E}\right)$-module. A cuspidal $G\left(\mathbf{A}_{t}\right)$-module $\pi$ is called distinguished if there is an integrable function $\varphi$ in the space of $\pi$ such that the integral $B(\varphi)=\int \varphi(x) d x$, on the closed subset $G(F) \backslash G(\mathbf{A})$ of $G(E) \backslash G\left(\mathbf{A}_{E}\right)$, is nonzero.

Let $M$ be a simple algebra of dimension $n^{2}$ central over $F$, where $n \geqslant 2$. Then there is a division algebra $D$ of rank $d$ dividing $n$ central over $F$ so that $M$ is the matrix algebra $M(m, D)$ of $m$ by $m$ matrices over $D$, where $n=d m$. Let $G$ be the quotient of the multiplicative group of $M$ by its center. Let $S$ be the set of places $v$ of $F$ where $D$ is ramified. Assume that each $v$ in $S$ splits in $E$. Let $G^{\prime}$ be the quotient of $\operatorname{GL}(n)$ by its center. For each place $v$ of $F$ we write $F_{v}$, for the completion of $F$ at $v$, and $E_{v}=E \otimes_{F} F_{l}$. For each $v$ outside $S$ we have $G_{v} \simeq G_{v}^{\prime}$, where $G_{v}=G\left(F_{v}\right), G_{v}^{\prime}=G^{\prime}\left(F_{v}\right)$. Fix two places $u$ and $u^{\prime}$ of $F$ with $u^{\prime}$ in $S$. Denote by $u$ and $u^{\prime}$ also a fixed place of $E$ above $u$ and $u^{\prime}$. Let $\pi$ be a cuspidal $G\left(\mathbf{A}_{t}\right)$-module which corresponds (by the Deligne-Kazhdan correspondence (see [F])) to a cuspidal $G^{\prime}\left(\mathbf{A}_{E}\right)$-module $\pi^{\prime}$, whose component $\pi_{u}^{\prime}$ at $u$ is supercuspidal, and at $u^{\prime}$ it is discrete-series. Thus $\pi_{r} \simeq \pi_{v}^{\prime}$ via $\bar{G}_{v^{\prime}} \simeq \bar{G}_{v}^{\prime}$, for any place $v$ outside $S$. We put $\bar{G}_{v}=G\left(E_{v}\right), \bar{G}_{n}^{\prime}=G_{v^{\prime}}^{\prime}\left(E_{v^{\prime}}\right)$.

THEOREM. $\pi$ is distinguished if and only if $\pi^{\prime}$ is distinguished.

The case of $n=2$, where $\pi_{u}^{\prime}$ is assumed to be only discrete-series, is due to Jacquet and Lai $[\mathbf{J L}]$. For general $n$ the condition at $u$ can be removed on applying furthur computations of the trace formula; but this will not be discussed here. The integrals $B(\varphi)$ were first studied by Shimura and Asai [A]. Applications of distinguished representations to the theory of Euler products are given in $\left[\mathbf{F}^{\prime}\right]$.

Received by the editors June 1, 1985 and, in revised form. May 1, 1986.

1980 Mathematics Subject Classification (1985 Revision). Primary 11F70.

1987 American Mathematical Society $0002-9939 / 87 \$ 1.00+\$ .25$ per page 
2. For every place $v$ of $F$ put $G_{v}=G\left(F_{v}\right)$, and let $R_{v}$, be the ring of integer in $E_{v}$. For $v$ outside $S$ put $K_{v}^{\prime}=G\left(R_{v}\right)$ and $K_{v}=K_{v}^{\prime} \cap G_{v}$, and let $f_{v}{ }^{\prime}$, be the quotient by the volume $\left|K_{n}\right|$ of the characteristic function of $K_{r}^{\prime}$. Fix a differential form of highest weight defined over $F$, hence a product measure $d x=\otimes d x_{\text {r }}$ on $G(\mathbf{A})$, so that the product of the volumes $\left|K_{l}\right|$ converges. Let $f=\otimes f_{l}$, be a function on $G\left(\mathbf{A}_{E}\right)$ such that for every place $v$ the component $f_{v}$, is a complex valued compactly supported function on $\bar{G}_{t}=G\left(E_{v}\right)$, which is locally constant if $v$ is finite, or smooth if $v$ is archimedean, with $f_{v}=f_{l^{\prime}}{ }^{\prime}$ for almost all $v$. Since $G^{\prime}$ is an inner form of $G$ we obtain a matching measure on $G^{\prime}(\mathbf{A})$, also denoted by $d x$. We choose a function $f^{\prime}=\otimes f_{1}^{\prime}$, on $G^{\prime}\left(\mathbf{A}_{E}\right)$, with the above properties, so that (1) at any $v$ outside $S$ we take $f_{v}^{\prime}=f_{v}$, via $\bar{G}_{v}^{\prime} \simeq \bar{G}_{v},(2)$ at any $v$ in $S$ which splits into $v^{\prime}, v^{\prime \prime}$ in $E$, the convolutions $h_{v^{\prime}}^{\prime}=f_{v^{\prime}}^{\prime *} * f_{v^{\prime \prime}}^{\prime \prime}$ and $h_{v}=f_{v^{\prime}}^{*} * f_{v^{\prime \prime}}$ have matching orbital integrals. Here $f_{t^{\prime}}^{*}(g)=f_{l^{\prime}}\left(g^{-1}\right)$,

$$
\left(f_{v^{\prime}}^{*} * f_{v^{\prime \prime}}\right)(g)=\int f_{v^{\prime}}^{*}\left(g x^{-1}\right) f_{v^{\prime \prime}}(x) d x,
$$

and our requirement is that for any regular $x$ in $G_{v^{\prime}} \simeq G_{v^{\prime}} \simeq G_{l^{\prime \prime}}$, and $x^{\prime}$ in $G_{v}^{\prime}$, which have the same sets of eigenvalues, we have

$$
\int h_{n}^{\prime}\left(g^{-1} x^{\prime} g\right) d g=\int h_{n}\left(g^{-1} x g\right) d g .
$$

The integrals are over $Z_{v}^{\prime}(x) \backslash G_{v}^{\prime}$, and $Z_{v}(x) \backslash G_{v}$, where $Z_{v}(x)$ is the centralizer of $x$ in $G_{v}$, and the isomorphic tori $Z_{v}(x)$ and $Z_{v}^{\prime}(x)$ are given matching measures.

Further, at some finite place $u$ of $E$ we require $f_{u}^{\prime}$, and $f_{u}$, to be supercuspidal. Namely, for any $E_{u}$-parabolic subgroup of $G_{u}^{\prime}$ with unipotent radical $N_{u}$, and any $x$, $y$ in $G_{u}^{\prime}$, the integral $\int f_{u}^{\prime}(x n y) d n$ is 0 . Hence the operator $r^{\prime}\left(f^{\prime}\right)=\int f^{\prime}(x) r^{\prime}(x) d x$ ( $x$ in $G^{\prime}(E) \backslash G^{\prime}\left(\mathbf{A}_{E}\right)$ ) on $L\left(G^{\prime}\right)$ is an integral operator with kernel $K^{\prime}(x, y)=$ $\sum_{\gamma} f^{\prime}\left(x^{-1} \gamma y\right)\left(\gamma\right.$ in $\left.G^{\prime}(E)\right)$.

An element $\gamma$ of $G^{\prime}(E)$ is called regular if it has distinct eigenvalues, and elliptic if it lies in a compact torus of $G^{\prime}\left(\mathbf{A}_{E}\right)$. Thus $\gamma$ is elliptic regular if it lies in no proper $E$-parabolic subgroup of $G^{\prime}(E)$. We say that $\gamma$ in $G^{\prime}(E)$ is relatively regular (resp. elliptic) if $\sigma(\gamma) \gamma^{-1}$ is regular (resp. elliptic) in $G^{\prime}(E)$. We denote by $\sigma$ the nontrivial element of $\operatorname{Gal}(E / F)$. Note that the centralizer of a regular $\sigma(\gamma) \gamma^{-1}$ is defined over $F$. From now on we deal only with relatively regular elements $\gamma$ in $G^{\prime}(E)$.

Lemma. Let $A$ be either a torus or a parabolic subgroup of $G^{\prime}$ defined over $F$. Then $\gamma$ lies in $A(E) G^{\prime}(F)$ if and only if $\sigma(\gamma) \gamma^{-1}$ lies in $A(E)$.

Proof. Suppose $A$ is parabolic over $F$. Then the first cohomology group $H^{1}(\mathrm{Gal}(E / F), A(E))$ is trivial. If $p=\sigma(\gamma) \gamma^{-1}$ lies in $A(E)$, we have $p \sigma(p)=1$. The cocycle $a_{\sigma}=p$ then splits, namely there is $u$ in $A(E)$ with $p=\sigma(u) u^{-1}$. Hence $g=\sigma\left(u^{-1} \gamma\right)=u^{-1} \gamma$ lies in $G^{\prime}(F)$, and $\gamma=u g$ is in $A(E) G^{\prime}(F)$.

Suppose $p$ is elliptic regular. Let $\theta$ be an element of $E-F$ with $\theta^{2}$ in $F$. Then $E=F(\theta)=F(\theta+c)$ for all $c$ in $F$. Put $\gamma=\gamma_{1}+\theta \gamma_{2}$, for $\gamma_{i}$ in $G^{\prime}(F)$. Consider the polynomial $P(c)=\operatorname{det}\left(\gamma_{1}+c \gamma_{2}\right)$. If $P(c)=0$ for all $c$ in $F$ then $P$ is identically zero, and $P(\theta)=0$, contrary to the assumption that $\operatorname{det} \gamma \neq 0$. Hence 
there is $c$ in $F$ so that $\gamma_{1}^{\prime}=\gamma_{1}+c \gamma_{2}$ is invertible. Put $\theta^{\prime}=\theta-c$. Then $\gamma=\gamma_{1}^{\prime}+$ $\theta^{\prime} \gamma_{2}$. Define $\delta=\gamma \gamma_{1}^{\prime-1}$, and $\delta^{\prime}=\gamma_{2} \gamma_{1}^{\prime-1}$. Then $\delta=1+\theta^{\prime} \delta^{\prime}$. If $\delta^{\prime}$ is nonelliptic in $G^{\prime}(F)$, then it lies in a parabolic subgroup of $G^{\prime}(F)$, hence $\delta$ lies in a parabolic of $G^{\prime}(E)$ defined over $F$, and so does $p=\sigma(\delta) \delta^{-1}$. contrary to our assumption. Hence $\delta^{\prime}$ lies in an elliptic torus $T(F)$ of $G^{\prime}(F), \delta$ lies in $T(E)$, and so does $p$. But $\delta$ commutes with the regular $p$, whose centralizer in $G^{\prime}(E)$ is $T(E)$. The Lemma follows.

We say that $\gamma$ and $\gamma^{\prime}$ in $G^{\prime}(E)$ are relatively conjugate if there are $x, y$ in $G^{\prime}(F)$ with $\gamma^{\prime}=x \gamma y$; equivalently (by the Lemma), if $\sigma(\gamma) \gamma^{-1}$ and $\sigma\left(\gamma^{\prime}\right) \gamma^{\prime-1}$ are conjugate by an element of $G^{\prime}(F)$. Indeed, by the Lemma we can assume that both $\gamma$ and $\gamma^{\prime}$ lies in $A(E)$.

Let $\{T\}$ be a set of representatives for the conjugacy classes of elliptic tori in $G^{\prime}(F)$. Put $\cup_{T} T(E) / \sim$ for the quotient of the relatively regular subset of $\bigcup_{T} T(E)$ by the equivalence relation: $t$ in $T(E)$ is equivalent to $t^{\prime}$ in $T^{\prime}(E)$ if $T=T^{\prime}$, and there is $w$ in the normalizer of $T(F)$ in $G(F)$, and $t^{\prime \prime}$ in $T(F)$, with $t^{\prime \prime}=t^{\prime} t^{\prime \prime}$.

COROllary. (1) $\gamma$ in $G^{\prime}(E)$ is relatively elliptic if and only if it lies in $G^{\prime}(F) T(E) G^{\prime}(F)$, where $T$ is an elliptic torus of $G^{\prime}$ defined over $F$.

(2) $\cup_{T} T(E) / \sim$ is a set of representatives for the relative conjugacy classes of the relatively elliptic regular elements in $G^{\prime}(E)$.

Assume that $f^{\prime}$ is such that $f^{\prime}(x \gamma y)$ is 0 for any $x, y$ in $G^{\prime}(\mathbf{A})$ and $\gamma$ in $G^{\prime}(E)$, unless $\gamma$ is relatively elliptic regular. Assume that $f$ satisfies the analogous condition. For example, we can make the analogous local assumption on the components $f_{u}^{\prime \prime}$ and $f_{u^{\prime}}$ at a place $u^{\prime}$ of $S$.

3. Proposition. We have $\iint K(x, y) d x d y=\iint K^{\prime}\left(x^{\prime}, y^{\prime}\right) d x d y ; x, y$ range over $G(F) \backslash G(\mathbf{A}) ; x^{\prime}, y^{\prime}$ are over $G^{\prime}(F) \backslash G^{\prime}(\mathbf{A})$.

Proof. The map which associates to $g$ in $\operatorname{GL}\left(n, \mathbf{A}_{E}\right)$ the coefficients $\left\{a_{i}\right.$; $\left.a_{n}=\operatorname{det} g\right\}$ in the characteristic polynomial of $g$ yields an isomorphism from the variety of semisimple conjugacy classes in $G\left(\mathbf{A}_{E}\right)$ to the quotient of $\mathbf{A}_{E}^{n-1} \times \mathbf{A}_{E}^{\times}$by $\mathbf{A}_{E}^{\times}$, where $\left\{a_{i}\right\} \simeq\left\{a_{i} z^{i}\right\}\left(z\right.$ in $\left.\mathbf{A}_{E}^{\times}\right)$. Suppose $f\left(x^{-1} \gamma x\right) \neq 0(\gamma$ in $G(E) ; x, y$ in $G(\mathbf{A}))$. Then the image of $x^{-1} \sigma(\gamma) \gamma^{-1} x$ lies in a compact subset of $\mathbf{A}_{E}^{n-1} \times \mathbf{A}_{E}^{\times} / \mathbf{A}_{E}^{\times}$, and also in the discrete subset $E^{n-1} \times E^{\times} / E^{\times}$, hence in a finite set. Consequently, only finitely many relative conjugacy classes (of relatively elliptic regular) $\gamma$ contribute to the sum $\sum f\left(x^{-1} \gamma x\right)$ over $\gamma$ in $G(E)$, which defines $K(x, y)$.

Replacing $f$ by its absolute value, we see that the integrals below are absolutely convergent; hence the rearrangements below are justified. Then

$$
\begin{aligned}
K(x, y) & =\sum_{T} \sum_{\gamma \in T(E)}^{\prime} \sum_{\alpha \in G(F) / T(F)} \sum_{\beta \in N(T) \backslash G(F)} f\left(x^{-1} \alpha \gamma \beta y\right) \\
& =\sum_{T} w(T)^{-1} \sum_{\gamma \in T(E) / T(F)}^{\prime} \sum_{\alpha \in G(F)} \sum_{\beta \in T(F) \backslash G(F)} f\left(x^{-1} \alpha \gamma \beta y\right),
\end{aligned}
$$

where $N(T)$ is the normalizer of $T(F)$ in $G(F), w(T)$ is the cardinalilty of its Weyl group, and the prime over $\sum$ indicates summation over relatively regular elements 
only. Integrating over $x, y$ in $G(F) \backslash G(\mathbf{A})$, we obtain

$$
\iint K(x, y) d x d y=\sum_{T} \frac{|T(\mathbf{A}) / T(F)|}{w(T)} \sum_{\gamma \in T(E) / T(F)}^{\prime} \iint f(x \gamma y) d x d y .
$$

On the right $x$ ranges over $G(\mathbf{A})$, and $y$ over $T(\mathbf{A}) \backslash G(\mathbf{A}) .|T(\mathbf{A}) / T(F)|$ is the volume of the compact group $T(\mathbf{A}) / T(F)$.

Each of the integrals is a product of local integrals. If $v$ is a place of $F$ which does not split in $E$ and $G_{v}=G\left(F_{v}\right), T_{v}=T\left(F_{l}\right)$, we obtain

$$
\iint f_{n}(x \gamma y) d x d y \quad\left(x \text { in } G_{l,}, y \text { in } T_{n} \backslash G_{n}\right) .
$$

This converges. Indeed, if the integrand is nonzero, then $x \gamma y$ lies in a compact, hence $y^{-1} \sigma\left(\gamma^{-1}\right) \gamma y$ is in a compact, hence $y$ is in a compact modulo $T\left(E_{v}\right)$ (since $\sigma\left(\gamma^{-1}\right) \gamma$ is regular), hence $y$ is in a compact modulo $T_{2}$. But for such $y$ the function $x \mapsto f_{r}(x \gamma y)$ is compactly supported, and our integral converges.

At almost all nonsplit $v$ we have that $f_{l}=f_{l}^{0}$ is the quotient by $\left|K_{l}\right|$ of the characteristic function of $K_{v}^{\prime}, E_{v} / F_{v}$, is unramified and $\gamma$ is in $K_{v}^{\prime}$. If $f_{v}(x \gamma y) \neq 0$ then $x \gamma y$ is in $K_{v}^{\prime}$, and so is $y^{-1} \sigma\left(\gamma^{-1}\right) \gamma y$. But $\sigma\left(\gamma^{-1}\right) \gamma$ is regular in $K_{v}^{\prime}$. Hence $y$ lies in $T\left(E_{v}\right) K_{v}^{\prime} \cap G_{v}$; since $E_{v} / F_{v}$, is unramified, the intersection is $T_{v} K_{v}$. Hence we can take $y$ in $K_{v}$, and conclude that $x$ is in $K_{v}$. Hence the integral is equal to the volume $\left|K_{v}\right| /\left|K_{v} \cap T_{v}\right|$ for almost all $v$ which do not split $E / F$.

If $v$ is a place of $F$ which splits into $v^{\prime}$ and $v^{\prime \prime}$ in $E$, then $\gamma=\left(\gamma^{\prime}, \gamma^{\prime \prime}\right)$ in $G\left(E_{n}\right)=G_{v} \times G_{v}$, and our local integral is

$$
\iint f_{r^{\prime}}\left(x \gamma^{\prime} y\right) f_{r^{\prime \prime}}\left(x \gamma^{\prime \prime} y\right) d x d y \quad\left(x \text { in } G_{r}, y \text { in } T_{r} \backslash G_{r}\right) .
$$

That is

$$
\iint f_{v^{\prime}}(x) f_{v^{\prime \prime}}\left(x y^{-1} \gamma^{-1} \gamma^{\prime \prime} y\right) d x d y=\int h_{v^{\prime}}\left(y^{-1} \delta y\right) d y,
$$

where $h_{v^{\prime}}=f_{v^{\prime}}^{*} * f_{v^{\prime \prime}}$, and $\delta=\gamma^{-1} \gamma^{\prime \prime}=\gamma^{-1} \sigma(\gamma)$ (we embed $G_{v}$ diagonally in $G\left(E_{v}\right)$ ). This is the orbital integral of $h_{v}$ at (the regular element) $\delta$, hence it converges.

For almost all such $v, f_{v^{\prime}}$ and $f_{v^{\prime \prime}}$ are the characteristic functions of $K_{v}$, divided by $\left|K_{l}\right|$, and $\gamma^{\prime}, \gamma^{\prime \prime}$ lie in $K_{v}$. If the integrand is nonzero, then $x \gamma^{\prime} y, x \gamma^{\prime \prime} y$ are in $K_{v}$. Hence $y^{-1} \delta y$ is in $K_{v}$, and $y$ is in $T_{v} K_{v}$. Taking $y$ in $K_{v}$, it follows that $x$ is in $K_{v}$, and the integral is equal to $\left|K_{v}\right| /\left|T_{v} \cap K_{v}\right|$ once again.

These computations hold for any reductive group $G$, in particular for our $G$ and $G^{\prime}$, and the equality of the proposition follows since we have the same sums, volume factors, and integrals on both sides. Indeed, at $v$ outside $S$ we take $f_{v}{ }^{\prime}=f_{v^{\prime}}$, and at $v$ in $S$ we take $f_{v}$, and $f_{v}^{\prime}$ so that $h_{v}$, and $h_{v}^{\prime}$, have matching (regular) orbital integrals, and this is precisely what is needed for the comparison of the local integrals.

4. Proof of theorem. Suppose $\pi$ is distinguished. We claim that so is $\pi^{\prime}$. The opposite direction is similar. Now if $v$ is a place of $F$ which splits into $v^{\prime}$ and $v^{\prime \prime}$ in $E$, then the restriction of $B$ to the space of $\pi$ is a nonzero $G(\mathbf{A})$-invariant form, 


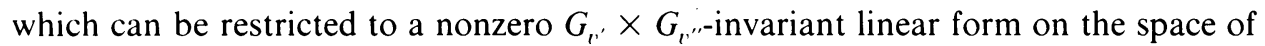
$\pi_{r^{\prime}} \times \pi_{r^{\prime \prime}}$; hence $\pi_{r^{\prime \prime}}$ is the contragredient of $\pi_{r^{\prime}}$, and we write $\pi_{r^{\prime}}$ for $\pi_{v^{\prime}}$, so that $\pi_{r^{\prime \prime}}=\check{\pi}_{r^{\prime}}$.

Let $S^{\prime}$ be a sufficiently large set of places of $F$ containing $S$ and the archimedean places, so that the space of $\pi$ contains a nonzero vector invariant under the action of $K^{S^{\prime}}=\prod_{l^{\prime}} K_{l^{\prime}}^{\prime}\left(v\right.$ outside $\left.S^{\prime}\right)$. For $v$ outside $S^{\prime}$ we take a $K_{l^{\prime}}^{\prime}$-bi-invariant $f_{v^{\prime}}$. Then $\pi^{S \prime}\left(f^{S^{\prime}}\right)$ factors through a projection from the space $V$ of $\pi$ to the space $V(\pi)$ of $K^{s^{\prime}}$-fixed vectors in $V$, and it acts on $V(\pi)$ as a scalar. The space $V(\pi)$ is

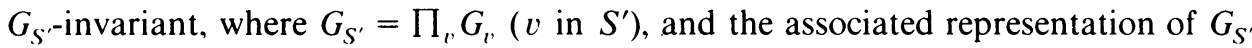
is denoted by $\pi_{S^{\prime}}$. Hence for $\varphi$ in $V(\pi)$ we have

$$
\pi(f) \varphi=\pi^{S^{\prime}}\left(f^{S^{\prime}}\right) \cdot \pi_{S^{\prime}}\left(f_{S^{\prime}}\right) \varphi .
$$

These comments apply to any cuspidal $G\left(\mathbf{A}_{E}\right)$-module $\rho$ with a supercuspidal component at $u$, so that we can write

$$
K(x, y)=\sum_{\rho} \rho^{s^{\prime}}\left(f^{s^{\prime}}\right) \cdot K_{\rho}(x, y),
$$

where $K_{\rho}$ is the kernel of $\rho_{S^{\prime}}\left(f_{S^{\prime}}\right)$, and the sum is over all cuspidal $G\left(\mathbf{A}_{E}\right)$-modules which contain a nonzero $K^{S^{\prime}}$-invariant vector, whose component at $u$ is supercuspidal. We have

$$
\iint K(x, y) d x d y=\sum_{\rho} \rho^{S^{\prime}}\left(f^{S^{\prime}}\right) a_{\rho}, \quad a_{\rho}=\iint K_{\rho}(x, y) d x d y .
$$

Note that $\pi_{S^{\prime}}=\otimes \pi_{1},\left(v\right.$ in $\left.S^{\prime}\right)$ is $\pi_{S} \otimes \pi_{S^{\prime \prime}}$, and $V(\pi)=V(S) \otimes V\left(S^{\prime \prime}\right)$, where $S^{\prime \prime}$ is the complement of $S$ in $S^{\prime}$, and $\pi_{S}=\otimes \pi_{l}, V(S)=\otimes V_{v}$, $(v$ in $S$ ); a similar definition holds for $S^{\prime \prime}$.

We may assume that there is a unitary vector $t$ in the space $V\left(S^{\prime \prime}\right)$, such that the restriction of $B$ to $t \otimes V\left(S^{\prime \prime}\right)$ is nonzero. Choose $f_{S^{\prime \prime}}$ so that $\pi_{S^{\prime \prime}}\left(f_{S^{\prime \prime}}\right)$ is the orthogonal projection on $t$. If $\left\{v_{k}\right\}$ is a basis of $V(S)$, then

$$
\iint K_{\pi}(x, y) d x d y=\sum_{k} B\left(t \otimes \pi_{S}\left(f_{S}\right) v_{k}\right) \bar{B}\left(t \otimes v_{k}\right) .
$$

Indeed,

$$
K_{\pi}(x, y)=\sum_{k} \pi_{S}\left(f_{S}\right) v_{k}(x) \bar{v}_{k}(y) .
$$

Note that if $u$ lies in $S^{\prime \prime}$ then we may assume that the component of $f_{S^{\prime \prime}}$ at $u$ is supercuspidal, since (1) if $g_{u}$ is supercuspidal then $f_{u} * g_{u} * f_{u}$ is supercuspidal, (2) if $\pi_{u}\left(f_{u}\right)$ is a projection on the space spanned by the unitary vector $t$, and $g_{u}(x)=$ $\left(t, \pi_{u}(x) t\right)$, then $\pi_{u}\left(f_{u} * g_{u} * f_{u}\right)=\pi_{u}\left(f_{u}\right) \pi_{u}\left(g_{u}\right) \pi_{u}\left(f_{u}\right)$ is also a projection on the space spanned by $t$, multiplied by a nonzero scalar.

The space $V(S)$ is the tensor product of the spaces $V_{l^{\prime}} \otimes V_{l^{\prime \prime}}$ over the $v$ in $S$, where $v^{\prime}, v^{\prime \prime}$ are the places of $E$ above $v$. The restriction of $B$ to $t \otimes V(S)$ is the tensor product of nonzero $G_{l} \times G_{l,}$-invariant linear forms $C_{l}$, on $V_{l^{\prime \prime}} \otimes V_{r^{\prime \prime}}$, up to a constant. For each $v$ in $S$ choose bases $\left\{a_{i}\right\}$ and $\left\{b_{j}\right\}$ of $V_{r}$, and $V_{r}$, which are dual to each other with respect to $C_{l}$. The sum over $k$ is equal (up to a constant) to

$$
\sum_{i j} C_{l^{\prime}}\left[\pi_{r^{\prime}}\left(f_{r^{\prime}}\right) a_{i} \otimes \pi_{r^{\prime \prime}}\left(f_{r^{\prime \prime}}\right) b_{j}\right] \bar{C}_{r^{\prime}}\left[a_{i} \otimes b_{j}\right] .
$$


As $C_{l,}\left(a_{i} \otimes b_{j}\right)=\delta_{i j}$ we obtain

$$
\begin{aligned}
& \sum_{i} C_{v^{\prime}}\left[\pi_{v^{\prime}}\left(f_{v^{\prime}}\right) a_{i} \otimes \check{\pi}_{l^{\prime}}\left(f_{v^{\prime \prime}}\right) b_{i}\right]=\sum_{i} C_{v^{\prime}}\left[\pi_{v^{\prime}}\left(f_{v^{\prime \prime}}\right) \pi_{v^{\prime}}\left(f_{v^{\prime}}\right) a_{i} \otimes b_{i}\right] \\
& =\sum_{i} C_{l^{\prime}}\left[\pi_{l^{\prime}}\left(f_{l^{\prime \prime}} * f_{l^{\prime}}\right) a_{i} \otimes b_{i}\right]=\operatorname{tr} \pi_{l^{\prime}}\left(f_{l^{\prime \prime}} * f_{l^{\prime}}\right) .
\end{aligned}
$$

If we replace $f_{r^{\prime \prime}}$ by $f_{r^{\prime \prime}}+f_{r^{\prime \prime}}^{*}$ we may assume that $f_{r^{\prime \prime}}^{*}=f_{r^{\prime \prime}}$ and that $h_{r}=f_{r^{\prime \prime}} * f_{r^{\prime}}$. It is clear that there is $h_{v}$, which is zero on the singular set, in fact supported on the elliptic regular set if $\pi_{l,}$ is discrete-series, so that $\operatorname{tr} \pi_{l,}\left(h_{l}\right) \neq 0$. We choose such a function at $u^{\prime}$.

Similar analysis applies in the case of $G^{\prime}$. In particular,

$$
\iint K^{\prime}(x, y) d x d y=\sum_{\rho^{\prime}} \rho^{S^{\prime}}\left(f^{\prime S^{\prime}}\right) a_{\rho^{\prime}}, \quad a_{\rho^{\prime}}=\iint K_{\rho^{\prime}}^{\prime}(x, y) d x d y,
$$

is equal to $\iint K(x, y) d x d y$ by the Proposition, for a function $f^{\prime}$ related to $f$ as there. Applying linear independence of characters of the Hecke algebra of $G^{S^{\prime}} \simeq G^{\prime S^{\prime}}$, we conclude that $\pi^{S^{\prime}}\left(f^{S^{\prime}}\right)=\pi^{\prime S^{\prime}}\left(f^{\prime S^{\prime}}\right)$ for our corresponding $\pi, \pi^{\prime}$, and that $a_{\pi^{\prime}}=a_{\pi}$ is nonzero. But $K_{\pi^{\prime}}^{\prime}$ is a sum of terms of the form $\varphi(x) \bar{\varphi}^{\prime}(y)$, where $\varphi, \varphi^{\prime}$ lie in the space of $\pi^{\prime}$. Hence the restriction of $B^{\prime}$ to the space of $\pi^{\prime}$ is nonzero, and $\pi^{\prime}$ is distinguished, as required.

\section{REFERENCES}

[A] T. Asai, On certain Dirichlet series associated with Hilbert modular forms and Rankin's method, Math. Ann. 226 (1977), 81-94.

[F] Y. Flicker, Representations of simple algebras, mimeographed notes, based on a course at Harvard University, fall 1985.

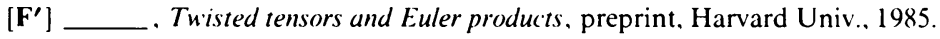

[JL] H. Jacquet and K. Lai, A relative trace formula, Compositio Math. 54 (1985), 243-310.

Dipartment of Mathematics, Princeton University, Princeton, New Jersey 08544 (Current address)

Dipartimint of Mathimatics, HaRvari) University, Cambridge, Massachusetts 02138 